\title{
BRAIN TUMORS CLASSIFICATION SYSTEM USING CONVOLUTIONAL RECURRENT NEURAL NETWORK
}

\author{
V.Akila ${ }^{1}$, P.K.Abhilash ${ }^{1}$, P Bala Venakata Satya Phanindra ${ }^{1}$, J Pavan Kumar $^{1}$, A.Kavitha ${ }^{2}$ \\ ${ }^{1}$ Gokaraju Rangaraju Institute of Engineering and Technology, Hyderabad \\ ${ }^{2}$ Vel Tech Rangarajan Dr.Sagunthala R\&D Institute of Science and Technology, Chennai
}

\begin{abstract}
The brain is a body organ that controls exercise of the relative multitude of parts of the body. Conceding robotized mind tumors in MRI (Magnetic Reverberation Imaging) is a confounded assignment given size and area variety. This strategy decides a wide range of malignancies in the body. Past techniques devour additional time with less accuracy. A manual assessment can be mistaken because of the degree of intricacies engaged with cerebrum tumors and their properties. However, the above proposition isn't appropriate for mind tumors because of colossal varieties in size and shape. Our proposed strategy to magnify arrangement performance. First, the expanded tumor district using picture enlargement is utilized to return for capital invested rather than the unique tumor area since it can give hints for tumor types. Second, expanded tumor locale split into progressively refined ring structure subregions. With three-component extraction approaches, employing photographs for information augmentation and rotating photographs at various angles, evaluate the performance of the suggested strategy on a large dataset. Utilizing Convolutional Recurrent Neural Network (CRNN), grouping of the tumor into three categories and thus give a virtual portrayal of exact value.
\end{abstract}

Keywords: Brain Tumour Classification; Convolutional Recurrent Neural Network; Image Classification; Deep Learning.

\section{Introduction}

Nowadays, electronic pictures in the clinical region are continuously being used for end. The prior ID of frontal cortex tumors is the fundamental key to treat the tumors effectively. Given the high fragile tissue separation and zero receptiveness to ionizing radiation, $\mathrm{X}$-beam is the unique technique for diagnosing human psyche tumors. In any case, frontal cortex tumor request is authentically not a little endeavor. The standard methodology for Xbeam mind tumor ID and gathering is by a human appraisal, which depends unequivocally upon the experience of radiologists who survey and separate the characteristics of pictures. Also, the director helped arrange strategies that are outlandish for a great deal of data and are non-reproducible. As such, PC upheld investigation instruments are especially appealing to determine these issues. Employments of frontal cortex tumor game plan can be essentially isolated into two classes: describing mind pictures into regular and weird classes, i.e., whether or not the frontal cortex pictures contain tumors; portrayal inside strange brain pictures; this way, isolation between 3 specific kinds of psyche tumors [1].

Tumors create because of the uncontrolled development of cells in mind. It can likewise be caused by malignancy in different body parts, which spreads to the mind. Cerebral pain, vision issues, and a slow loss of sensation are the usually noticed manifestations. Therapy relies upon the size and area of the tumor; medical procedure, radiation treatment, and chemotherapy might be recommended. In the current examination, we center around the order of three sorts of mind tumors (i.e., meningioma, glioma, pituitary tumor) in $\mathrm{T} 1$ weighted difference - upgraded X-ray images. Spatial Pyramid Matching (SPM) coordinating parts picture into progressively refined rectangular subregions and registers histograms of the nearby element from each sub locale, making magnificent outcomes for regular scene characterization. Fair treatment, arranging, and exact diagnostics ought to be carried out to improve the patients' future. The best strategy to distinguish mind tumors is Attractive Reverberation Imaging (X-ray) [2]. A gigantic measure of picture information is produced through the outputs. The radiologist inspects these pictures.

Feature extraction is a fundamental development in portrayal as additional illuminating features will undoubtedly improve request accuracy. In various past assessments, power and surface features, similar to firstdemand bits of knowledge, Gabor channels, and wavelet change, are the most intermittently used methods to 
portray mind tumor pictures. Jiang et al. proposed a 3D voxel request-based frontal cortex tumor division method using CNN classifier [9]. Recently, the improvement of artificial intelligence estimations at picture plan affirmation tasks has been growing quickly, promising results in aiding the clinical planning field. With the upheld progression of clinical imaging methodology, there has been an improvement in the precision and steadfastness of clinical findings. Regardless, a PC-supported discovery can diminish the end time and will be valuable in zeroing in on the reports[10]. Continuous advances in artificial intelligence have shown that the customized picture gathering using estimations can perceive channels in a CT inspection. In particular, Significant learning has shown promising results in automated plans. It is so proficient at picture tasks that we can use neural associations to make logical pictures, not just separate them. Especially CRNN Association is fitting for analyzing pictures, for instance, CT or X-beam channels. CRNNs are developed generally to deal with pictures even more adequately and complete picture groupings. This way, CRNNs approximates radiologists' precision while perceiving critical features in CT channels or some other characteristic pictures [3].

The significant learning computations could robotize the end method for requesting CT check pictures. Therefore, encouraged a CRNN model to arrange CT check pictures subject to its features. This will help reduce the time in the channel end and could, in like manner, be valuable in zeroing in on the radiologist worklist. Additionally, it might be helpful in regions where an expert radiologist is distant and helps understudies. However, there are fewer transparently available datasets, and there is a prerequisite for more datasets besides improving the work.

The CRNN designing produces convolutional layers, the pooling layer, and the related layer and stacks them together. It can make many convolutional and pooling layers. More the layers, more the features eliminated. The convolutional layer is created with $3 * 3$ size and considering the 'relu' authorization work. Later on, both the layers are stacked, and a related layer is created with softmax sanctioning. The softmax layer figures the softmax, saving a considerable load of time and improving security. It takes two wellsprings of information. First, it considers the assumption for the last layer and the second one as the name layer. Second, it processes the hardship work used by the backpropagation estimation to figure tendencies concerning loads in the network [7]. Along these lines, our model designing is ready. As of now, the model will take incorporate and get arranged. Python is a programming language that proposes a language the two individuals and PCs can appreciate. Python was made by a Dutch designer named Guido van Rossum, who made the language to manage explicit issues he found in coding of the time.

Python is an unraveled critical-level programming language, for the most part, necessary programming.
Made by Guido van Rossum and first passed on 1991, Python has a plan believing that nerves code clarity and a semantic program that licenses specialists to bestow considerations in fewer lines of code, strikingly utilizing fundamental whitespace. It gives cultivates that empower straightforward programming on both little and massive degrees.

Python consolidates a unique sort structure and redid memory the board. It keeps up different programming ideal models, including object-masterminded, essential, meaningful, and procedural, and has a colossal and careful standard library.

Python go-betweens are open to some working frameworks. C Python, the reference execution of Python, is open-source programming and has a local improvement model, as accomplished in every practical sense, its assortment executions. The non-advantage Python Programming Establishment regulates C Python.

Previous methods consume more time and less accuracy. Using the original tumor region, we get considerable variations in size and shape. Here, MRI splits into increasingly acceptable rectangular and computes histograms of local features from each subregion.

The programmed cerebrum tumor location of a patient comprises two significant stages: specific, picture division, and edge discovery. The following considerable advance is adding way from the picture informational collection record to the framework index. It tends to be executed by a boa constrictor outline work to designate informational indexes from PC memory. However, it may be performed either physically or by programming utilizing the python operating system inbuilt activity.

\section{Literature survey}

In the literature, various computations and different changes of the pre-arranged associations are used for picture assessment, course of action, and division. Different procedures have been taken a stab at other clinical data puts together, both on X-beam pictures of brain tumors and tumors from various human body segments. These papers were not considered further, as the accentuation was on the documents using a comparable X-beam picture database. The decision is to use all of the open planes could increase the database. As this could all-around impact the course of action yield by overfitting, pre-getting ready is required preceding dealing with the photos into the neural association.

Regardless, one of the known advantages of Convolutional Neural Network (CNN) associations is that the pre-planning and the part planning should not be performed. Taking everything into account, the best result in the composing using the separated picture parts as information sources are presented by Tripathi and Sack, with $94.64 \%$ precision. For commitment to the classifiers, they use features eliminated from the image's divided frontal cortex. In addition, they attempted their 
strategy using a 5-wrinkle cross-endorsement technique.

A relationship with the same state-of-the-art methods shows that our association got better results. The best outcome for 10-overlay cross-endorsement was refined for the record-wise procedure additionally, for the extended dataset, and the exactness was $96.56 \%$. Taking everything into account, in the composition, no paper shows attempted hypothesis, through the subject-wise koverlay method, for this informational image index. For the subject-wise procedure, we gained a precision of $88.48 \%$ for the expanded dataset. The typical test execution was under $15 \mathrm{~ms}$ for each image. These results show that our association has a good theory capacity and incredible execution speed. It might be used as a practical decision help instrument for radiologists in clinical diagnostics.

\section{Convolutional recurrent neural network}

Image classification preferably need to characterize a picture regardless of its size, position, direction, immersion, splendor, and numerous other outer variables. This is the place where Neural Organizations can take care of us. Profound neural organizations give best outcomes than any remaining existing strategies due to their different highlights like removing covered-up highlights, equal handling, and constant activity. The philosophy of profound neural organizations, particularly convolutional neural organizations, closely resembles that of the association of neurons in the human mind. Singular neuron reacts to improvements just in a confined district known as Responsive Field [2]. CRNN (ConvNet) is a profound learning calculation that accepts pictures as info and can separate one from another. It brings the spatial and fleeting conditions in a picture. This engineering acts well for picture datasets as the number of external variables is decreased prior to taking care of the pictures to the design. ConvNet changes over pictures into a structure that is simpler to measure. It additionally takes care that the fundamental highlights are not lost as they assume a significant part in classification [5]. Pictures are perused as pixels by the PC. CNN accepts each picture as info and structures many hidden layers prior to preparing the yield. The principal focal point of CRNN lies in removing the undeniable level highlights like edges, colors, inclinations, directions, and so on. There is no specified principle that ConvNet should restrict to just one layer. In any case, the primary layer is liable for separating low-level highlights. In the progressive layers, each layer continues extricating the undeniable level highlights individually, at long last giving us an organization. The convolved highlights can be diminished or expanded dimensionality by applying legitimate padding [4].

Pooling is another virtual device that CRNN employments. Like the ConvNet layer, the pooling layer also helps in lessening the spatial size while safeguarding the absolute highlights of the picture. The math associated with pooling is moderately straightforward. It keeps up the most significant worth from every window at each progression. The thought is left with about a quarter of however many pixels as it began with. This decreases the computational ability to deal with the information through dimensionality decrease, which is the main benefit of CNN. Conventional calculations set aside more effort for highlight extraction and preparing. The convolutional layer and pooling layer structure each ith layer of the convolutional neural network. Contingent upon the picture, the quantity of layers continues to change. This interaction effectively empowers the model to comprehend the highlights of the image in the datasets. Afterward, level the last yield and feed it to a customary neural organization for characterization.

\subsection{CRNN model architecture}

The CRNN design creates convolutional layers, the pooling layer, and the completely associated layer and stacks them together [6]. It can make quite a few convolutional and pooling layers. More the layers, more the highlights removed. The convolutional layer is created with $3 * 3$ size and considering the 'relu' initiation work. Later on, both the layers are stacked, and a wholly associated layer is produced with softmax enactment. The softmax layer computes the softmax, saving a ton of time and improving strength. It takes two sources of information [8]. First, it considers the forecast of the last layer and the second one as the name layer. Second, it computes the misfortune work utilized by the backpropagation calculation to figure angles concerning loads in the network [5]. In this manner, our model design is prepared. Presently, the model will take include and get prepared. They are 39,341,894 params, out of which all are teachable, and there are zero nontrainable params. The picture below sums up the model engineering.

\subsection{About python}

Python is a programming language, which implies that it is a language that individuals and PCs can comprehend. Python was imagined by a Dutch programmer named Guido van Rossum, who made the language to tackle a portion of the issues he experienced in coding around then. Python incorporates an incredible program and programmed memory for the executives. It upholds numerous altering standards, including object-situated, fundamental, practical, and measure, and has an enormous and complete general library.

\subsection{Pandas}

The name Pandas name is gotten from the expression "board information," the monetary name of the informational collections in mass request. It is an information base for information the executives and examination. In addition, the library gives information constructions and usefulness to the administration of number tables and time arrangement. Otherwise called the "Python Information Investigation Library." 
NumPy is a standard rundown preparing bundle. It gives the most effective device to similar individuals and the devices to work with this rundown. Matplotlib is a python $2 \mathrm{D}$ altering library that produces print quality insights for an assortment of printed copy and intelligent stages. Matplotlib permits you to create destinations, histograms, power spectra, bar graphs, mistake outlines, Sciplots.

\subsection{Scikit-Learn}

Scikit-learn is an AI machine. It contains an assortment of arrangement, translating, and coordination calculations, including vector emotionally supportive networks, irregular woodlands, slope broadening, kmeans, and DBSCAN, and is intended to work with mathematical and logical libraries Python,NumPy and SciPy.

\subsection{Deep learning}

Profound learning is under AI (ML), which keeps on switching the world up to us. From non-mechanized vehicles to discourse acknowledgment, top to bottom learning makes it all conceivable. It has become an interesting issue for Industry and scholastics and influences practically all businesses identified with ML and Man-made consciousness (computer-based intelligence). "Profound learning (otherwise called efficient perusing or successive perusing) is essential for a more extensive group of AI strategies dependent on the portrayal of learning information, dissimilar to calculations explicit to a specific assignment. Learning can be administered, somewhat observed or directed".

The AI Branch wise worked utilizing enormous Fake Neural Organizations ( $>100$ layers). Preparing ANNs for profound learning requires a great deal of named information and a ton of registering power.

"Profound" likewise alludes to the many hidden layers of Artificial Neural Network (ANN) Organizations utilized in profound learning. There are various sorts of ANNs for different circumstances that will be talked about over the long haul. Deep learning has grown all the more precisely throughout the years and proceeds. Understanding its subtleties will help us all.

\subsection{Preprocessing}

CRNN is converting pictures into Clusters. The principle's testing task eliminates antiquities delivered by inhomogeneity in an attractive field or little developments made by the patient during filtering. Many times, predisposition is available in the checking results, which influence the division results, especially in the PC n-based models. Chmelik et al. showed work that utilizations predisposition adjustment for the $\mathrm{T} 1$ and $\mathrm{T} 1 \mathrm{C}$ pictures in the informational index. The $\mathrm{n} 4 \mathrm{~T} 1 \mathrm{~K}$ inclination remedy eliminates the power angle on each examining image.
Furthermore, clamor decrease is additionally performed by the central channel to normalize the pixel forces. Subsequently, clamor decrease and predisposition adjustment help improve the information handling and give better division; numerous radiofrequencies beat arrangements can be utilized to provide various tissue types. Four unique successions accessible for each picture in the Imps data set, similar to liquid weakened recovery compound and physiological attributes, can be acquired from these heartbeat arrangements, which bring about the differentiation between the individual classes. CRNN is converting pictures into Exhibits.

\subsection{Data augmentation}

Information increase incorporates a broad scope of methods to create "new" preparing tests from the first ones by applying irregular nerves and irritations. Our objective while applying information expansion is to expand the generalizability of the model as shown in Fig.1.
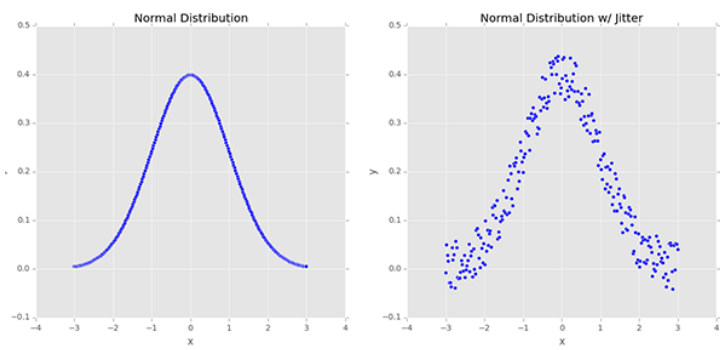

Fig.1: Sample Image Augmentation

\subsection{Training and testing}

As the model design is prepared, it can apply the neural organization model to the preparation dataset. Exactness is utilized as a measurement for estimating productivity. Age demonstrates the number of passes of the preparation dataset the calculation has finished. The worth is set to 10 . In any case, the period is not the same as emphasis. By and large, the majority of the models utilize more than one age. Just a single generation is used just when the accurate preparation information is the bunch size. Deciding the number of ages a model should rush to prepare will rely upon numerous boundaries identified with both the data and the model's objective. The preparation dataset precision continues expanding for every age, demonstrating that the model is gaining precisely from the information. The informational approval index is additionally tried, and the test information after the CNN model shows high precision. All the testing informative index is given as a contribution to the model and tracked down. The model can order the test pictures with suitable class marks with an exactness of $99.804 \%$, which conventional AI calculations can't accomplish as shown in Fig.2. 


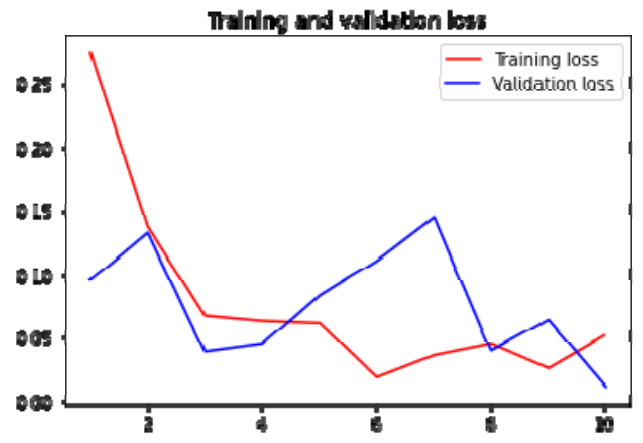

Fig.2. Training and Validation Loss

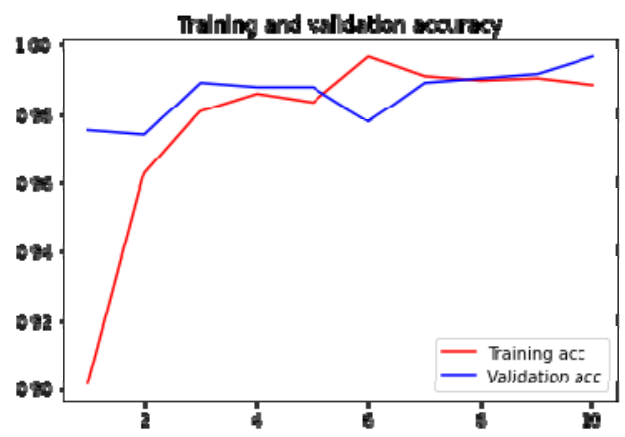

Fig.3. Training and Validation Accuracy

The $\mathrm{X}$-axis addresses the number of ages in the two charts. The Y-hub addresses the level of exactness and misfortune separately. The outcomes got are intriguing as they are portraying the presentation. The red bend addresses the bend for the preparation dataset as shown in Fig.3. The blue angle addresses a turn for the approval dataset. As the quantity of ages builds, preparing misfortune and testing misfortune is slowly diminished, a positive pointer for excellent execution as shown in Fig.4

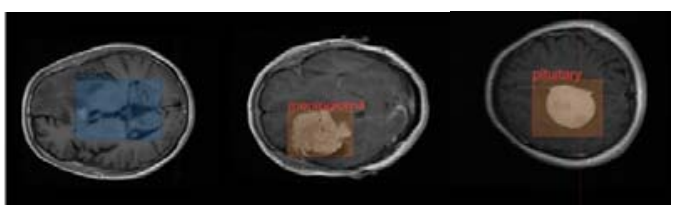

Glioma Meningioma Pituitary

Fig.4. Image of Glioma, Meningioma, Pituitary

\section{CONCLUSION}

The examination work portions cerebrum tumor into four classes like edema, non-improving tumor, upgrading tumor, and necrotic tumor. Mind tumor division needs to isolate solid tissues from tumor districts like propelling tumor, necrotic center, and encompassing edema. This is a fundamental advance in finding and treatment arranging, the two of which need to occur rapidly if there should arise an occurrence of harm to boost the probability of fruitful treatment. This classifier model fundamentally has fewer pictures in the dataset. In this way, even though the exactness is acceptable, the proficiency of the model should be as yet improved. Gathering a more excellent dataset can generally build the productivity of this model. What's more, the nature of pictures used to make the model can likewise impact the model. The entanglement with a clinical issue is the bogus negative and bogus positive tallies. Bogus negative, fundamentally in our concern, is a significant angle since this could prompt the patient's demise. To deal with this, we can irregularity the dataset to such an extent that more certain classes are in it. This may cause an expansion in bogus positive tally; however, bogus positive is only a burden to the patient, while bogus negatives could bring about death.

\section{References}

1. Wang J, Yang J, Yu K, Lv F, Huang T, Gong Y. (Proc IEEE Comput Soc Conf Comput Vis Pattern Recognit.,2010)

2. Liu W, He J, Chang S. (Proc 27th Int Conf Mach Learn., 2010)

3. Matas J, Chum O, Urban M, Pajdla T. (Br Mach Vis Conf. ,2002)

4. Prasanna Lakshmi K, Reddy CRK. (Int Conf. on Networking and Information Technology,2010)

5. Yang J, Yu K, Gong Y, Huang T. (IEEE Conf Comput Vis Pattern Recognit., 2009)

6. Dhanalaxmi B, Apparao Naidu, G., Anuradha.K (Procedia Computer Science.,2015)

7. Akila,V.,Sriharshini,K.,Sravani,P.,Sravanthi,D.,Go pi,R.,Sheela,T., International Journal of Online and Biomedical Engineering, 17(1),120-128 (2021)

8. Hussaini,S.M.,Krishna,G.,Gupta,A.K.,Singh,S.K.,J ournal of Manufactoring Processes, 18,151158(2015)

9. Sateesh,N.,SampathRao,P.,Ravishankar,D.V.,Satya narayana,K. ( Materials Today: Proceedings.,2015)

10. Hussaini, S.M., Singh, S.K., Gupta, A.K., Journal of Materials Research and Technology.,3(1),1724,2014 . 